\title{
Is there a future for cell-free fetal DNA tests in screening for preeclampsia?
}

\author{
Urszula Sarzynska-Nowacka, Przemyslaw Kosinski, Miroslaw Wielgos \\ First Department of Obstetrics and Gynaecology, Medical University of Warsaw, Poland
}

\begin{abstract}
CffDNA screening is a powerful diagnostic tool in the prenatal diagnosis algorithm for chromosomal abnormalities. With detailed ultrasound examination as the mainstay of first-trimester risk assessment, cffDNA has been shown to be superior to first-trimester combined screening (FTCS) in false-positive rates for trisomy 21 detection. In light of the growing interest in cffDNA testing and the possibility of it replacing first-trimester biochemistry, we decided to investigate the usefulness of cffDNA tests in early-pregnancy risk assessment for preeclampsia (PE). The aim of this review paper was to evaluate clinical application of first-trimester cfDNA in predicting $\mathrm{PE}$, as well as to investigate its possible use in first-trimester PE screening enhancement, also in cases where biochemistry is not performed.
\end{abstract}

Key words: cell-free fetal DNA; cffDNA; cell-free DNA; cfDNA; preeclampsia; PE; first-trimester screening; first-trimester combined screening

Ginekologia Polska 2019; 90, 1:55-60

\section{INTRODUCTION}

In the 1990s, Lo et al., published a revolutionary paper describing the presence of cell-free DNA (cfDNA) of fetal origin (cell-free fetal DNA; cffDNA) in maternal circulation, and reported its increased concentrations in pregnancies affected by trisomy 21 [1, 2]. Since its clinical implementation in 2011, cffDNA testing has been confirmed as a highly accurate non-invasive screening test for trisomy 21 among various groups of patients [3]. Currently, cfDNA screening is a powerful diagnostic tool in the prenatal diagnosis algorithm for chromosomal abnormalities. With detailed ultrasound examination as the mainstay of first-trimester risk assessment, cffDNA has been shown to be superior to first-trimester combined screening (FTCS) in false-positive rates for trisomy 21 detection [4]. In light of the growing interest in cffDNA testing and the possibility of it replacing first-trimester biochemistry, we decided to investigate the usefulness of cffDNA tests in early-pregnancy risk assessment for preeclampsia (PE).

Following the Fetal Medicine Foundation principles, cffDNA test serves as a screening tool in the intermediate risk group only since high-risk groups require invasive testing and low-risk patients are managed expectantly. Some authors have suggested an increased release of fetal DNA into the maternal bloodstream due to trophoblastic apoptosis in abnormal placentation disorders, such as PE or fetal growth restriction (FGR) [5]. PE complicates 2-3\% of all pregnancies and, together with its most malignant form - eclampsia, is responsible for a great number of maternal deaths globally [6], posing a formidable challenge for maternal-fetal medicine.

The aim of this paper was to review a potential role of cfDNA in maternal-fetal medicine with special regard for application of first-trimester cfDNA in predicting PE. The well-known and extensively described function in screening for trisomies was not included. It is believed that this article will provide insight into broader spectrum of cfDNA employment in diagnosis of pregnancy complication.

\section{Cell free fetal DNA and PE}

Recent meta-analyses and the results of randomized controlled ASPRE trials triggered a serious interest in first-trimester PE screening. A possibility of effective reduction in PE prevalence by early (before 16 weeks of gestation) implementation of aspirin has been recently well-described in the literature [7-9]. Although various studies report increased concentrations of cfDNA in women with confirmed PE, or shortly before the onset of $\mathrm{PE}$, there is paucity of data on the predictive value of cfDNA testing at first-trimester visit $[10,11]$. 
Increased quantities of fetal and total cfDNA in women diagnosed with PE are probably the result of enhanced apoptosis of the trophoblastic cells resulting from placental ischemia, inflammatory processes, and reduced clearance of cfDNA from the maternal circulation [12]. Paradoxically, lower fetal fraction might originate from disturbed ratio of released cfDNA, with a more pronounced increase in the maternal fraction.

\section{Technology behind cffDNA}

The initial detection of cffDNA was based on $Y$ chromosome-specific markers, narrowing the investigation to male-fetus pregnancies only. The discovery of an epigenetic disbalance in maternal and fetal genes has brought a new approach to quantification, based on polymerase chain reaction (PCR). The promoter of the RASSF1A gene, hypermethylated in the placenta and hypomethylated in maternal blood cells, seems to be the appropriate candidate for digestion with methyl-sensitive restriction enzymes, which allows to detect placental-derived sequences in the maternal circulation $[13,14]$. Despite the fact that this method might determine future directions for PE screening, risk should still be evaluated by means of non-PCR based first-trimester screening. Different methods widely used for commercial purposes include massively parallel shotgun sequencing (MPSS) and more selective methods, such as chromosome selective sequence analysis (CSS) or a single nucleotide polymorphism (SNP)-based analysis [15]. MPSS appears to be the most appropriate in surveying the content of maternal plasma DNA. MPSS works by analyzing millions or even billions of nucleic acids and comparing them with the reference sequence, which generates high costs. DNA molecules, acting as short fragments in human plasma, could be detected by commercial MPSS platforms, more efficient for analyzing highly fragmented DNA as compared to locus-specific methods of analysis [16]. With CSS or SNP methods, much less analysis is required since the assays are directed against specific regions, which significantly reduces the costs. Both methods are widely accepted as high-performance providers in cffDNA analysis, yet, to the best of our knowledge, there are no studies comparing the results in the same populations. An overview of the commercially available methods for cfDNA testing is presented in Table 1.

Commercial laboratories should present their methodology and clinical validation data in detail. Moreover, quality metrics must be given for reliable reporting. Fetal fraction measurement could be used for laboratory comparison - low failure rate might indicate inaccurate assessment of low fetal fraction, and high failure rate may be expected in overly complicated tests or those of low performance [15].

\section{Fetal fraction and maternal factors}

Associations between fetal fraction in maternal plasma and maternal characteristics were described extensively and most results are mutually comparable. Ashoor et al. [17] estimated median fetal fraction at 11-13 weeks of gestation to be $10 \%$. In their study, fetal fraction decreased with maternal weight, increased with fetal crown-rump length, maternal serum level of free beta-hCG (free beta-human chorionic gonadotropin) and PAPP-A (pregnancy-associated plasma protein-A). Moreover, it was higher in smokers and in the presence of fetal trisomy 21 . Ethnicity also plays a role in cffDNA fraction - women of Afro-Caribbean origin seem to have lower levels than Caucasians, yet the greatest depletion is provided by maternal weight - the estimated proportion of pregnancies with fetal fraction below $4 \%$ increased with maternal weight from $<1 \%$ at $60 \mathrm{~kg}$ to $>50 \%$ at $160 \mathrm{~kg}$ [17]. Similar results were reported by Revello et al. [18]. Fetal fraction decreased with growing BMI and maternal age and was also lower in women of South Asian origin than in Caucasians. It was higher in natural conception as compared to assisted reproduction techniques, and with higher levels of PAPP-A and beta-hCG. Median fetal fraction in the group unaffected by chromosomal trisomies was at the level of $11 \%$ (interquartile range, IR, $8.3 \%-14.4 \%$ ), $10.7 \%$ in trisomy $21,8.6 \%$ in trisomy 18 , and $7.0 \%$ in trisomy 13 . Fetal fraction concentration in relation to PE was also

\begin{tabular}{|c|c|c|c|}
\hline Method & MPSS & CSS & SNP \\
\hline Technique & $\begin{array}{l}\text { quantification of DNA fragments } \\
\text { originating from any given chromosome }\end{array}$ & $\begin{array}{l}\text { selective assays directed against specific } \\
\text { regions on chromosomes } 21,18,13, X, Y\end{array}$ & $\begin{array}{l}\text { analysis of differences in maternal and } \\
\text { fetal single nucleotides within a given } \\
\text { sequence }\end{array}$ \\
\hline Advantages & $\begin{array}{l}\text { + fragments from all chromosomes are } \\
\text { examined } \\
\text { + low failure rate }(<2 \%) \\
\text { + possible microdeletion/ } \\
\text { microduplication detection }\end{array}$ & + reduced cost compared to MPSS & $\begin{array}{l}\text { + triploidy detection } \\
\text { + possible extension to selected } \\
\text { microdeletion/ } \\
\text { microduplication offered by a company }\end{array}$ \\
\hline Disadvantages & - high cost & - failure rate of $2-4 \%$ & - failure rate of $3-5 \%$ \\
\hline
\end{tabular}

MPSS - massively parallel shotgun sequencing analysis; CSS — chromosome selective sequence analysis; SNP — selective nucleotide polymorphism analysis 
presented by Rolnik et al. [19]. First-trimester markers for preeclampsia (mean arterial pressure - MAP, uterine artery pulsatility index - UtAPI, PAPP-A and placental growth factor - PIGF) concentrations were analyzed alongside the baseline characteristics of the population. Gestational age as well as multiparity without history of PE or FGR had a positive association with fetal fraction. On the other hand, IVF conception, advanced maternal age, increased BMI, chronic hypertension, types 1 or 2 diabetes mellitus, and South Asian ethnicity were associated with diminished fetal fraction. Finally, MAP and UtAPI showed a substantial negative association with fetal fraction $(r=-0.118, p<0.001$ and $r=-0.064, p<0.001$ for MAP and UtAPI, respectively), and PAPP-A and PIGF had a significant positive association with fetal fraction $(r=0.219, p<0.001$ and $r=0.091, p<0.001$ for PAPP-A and PIGF, respectively).

According to the literature, gestational age and inversely correlated maternal weight are the only two factors strongly influencing fetal fraction [20]. Other variables, e.g. ethnicity, cigarette smoking or chromosomal aneuploidies, show confusing results and need further research [17].

\section{Low fetal fraction and the risk for preeclampsia}

The percentage of placental fraction in maternal circulation is crucial to obtain informative and reliable results. Poor accuracy is related to low fetal fraction of cffDNA [21]. Most companies avoid presenting their results if placental fraction is lower than $4 \%$. Approximately $2-3 \%$ of the results are inconclusive due to logistic failures (specimen collection, storage or processing), or genomic irregularities (fetal, maternal, placental mosaicism, malignancy, other chromosomal abnormalities), with insufficient fetal fraction as the most common reason [22].

Rolnik et al. [19], supported the theory that low fetal fraction might be an implication of diminished placental mass and, consequently, an early marker of placental dysfunction. A total number of 4313 singleton pregnancies which had undergone cfDNA testing at the first trimester visit were analyzed for PE or FGR risk. Their study was the first analysis of a correlation between first-trimester fetal fraction and biophysical markers for PE. Lower fetal fraction was associated with higher risk for PE $<34$ weeks, $<37$ weeks, FGR $<37$ weeks and correlated with higher MAP and UtAPI. On the other hand, fetal fraction was positively associated with PAPP-A and PIGF and could be partially predicted by maternal characteristics. Assuming a risk cut-off 1:100 for $\mathrm{PE}<37$ week gestation, median fetal fraction for the high-risk group was 10.4\% (IQR 7.8, 13.2), compared to 11.8\% (IQR 9.2, 14.8 ) in the low-risk group ( $p<0.001)$, which is consistent with the findings of another study with known pregnancy outcome $-11.04 \%$ in patients who did not developed PE as compared to $10.06 \%$ in PE patients ( $p<0.0001$ ) [23].
In that study by Norton et al., the association of UtAPI and PIGF with fetal fraction did not remain independently significant after addition of PAPP-A to the multivariate model, probably due to its strong own association with fetal fraction. The question remains whether fetal fraction incorporated to multi-marker screening would improve the accuracy of the test. Although its contribution may not be significant due to strong associations of PE with other well-described markers, it may play a role in cases without the results of placental biochemistry. The main limitation of the study by Norton et al., is lack of information whether women with low fetal fraction actually developed $P E$, although this observation probably could not have been made due to changes in the natural course of PE caused by aspirin implementation.

In a large pooled meta-analysis, Contro et al. [11], did not find usefulness of first-trimester cffDNA quantification for predicting PE measured by means of RT-PCR (reverse transcription polymerase chain reaction), a technique not used in commercially available cffDNA tests. A great strength of their review was the analysis of PE prediction not only in high-risk women but also those who subsequently developed PE. Still, these authors did not mention aspirin intake among their study population as a confounding factor. Despite a considerable number of reviewed papers, only two of them investigated cffDNA measured in the first trimester for PE prediction at any time during pregnancy (Tab. 2).

Based on data shown in Table 2, it seems that at present first-trimester cffDNA cannot be used as a predicting tool for $P E$, since DR is too low to be clinically significant. Moreover, when Sifakis et al. [24], took into account late PE (after 34 weeks of gestation), the difference between cffDNA levels became insignificant (all PE vs. controls - 71.2 vs. 51.5; late PE vs. controls - 50.8 vs. 51.5 ), which gives unacceptably low DR. In the study by Kim et al the DR rate was higher, although their study group was small - only 4 cases of PE. Despite many limitations of their analysis, the data were collected in a rigorous manner and merged into pooled analysis with recalculation of data distribution.

Papantoniou et al., [25] quantitated both, cfDNA and cffDNA by determining RASSF1A levels before and after enzyme digestion (3.0 - fold and 20.0 - fold elevation, respectively) in women who subsequently presented $\mathrm{PE}$, and referenced their results to uncomplicated pregnancies. In the PE group, RASSF1 A levels were significantly higher as compared to gestational-age-matched uncomplicated pregnancies (median cfDNA: $9402 \mathrm{gEq} / \mathrm{mL}$ vs $2698 \mathrm{gEq} / \mathrm{mL}$; $p=0.000 ; g E q=$ genome equivalents). After enzyme digestion and RASSF1A gene selective amplification, a 20-fold increase was observed (median cffDNA: $934.5 \mathrm{gEq} / \mathrm{mL}$ vs $62 \mathrm{gEq} / \mathrm{mL} ; \mathrm{p}=0.000$ ). Thereby, cffDNA fraction of cfDNA could be estimated $(2.16 \%$ and $14.4 \%$ in unaffected and $P E$ pregnancies, respectively). 
Table 2. Actual cffDNA values, estimated DR and AUC at a $10 \%$ false positive rate cffDNA in first-trimester screening

\begin{tabular}{|l|l|l|}
\hline & Author & \multicolumn{2}{l|}{} \\
\cline { 2 - 3 } & Kim et al. [35] & Sifakis et al. [24] \\
\hline Weeks & $7-14$ & $11-14$ \\
\hline No. of controls & 36 & 176 \\
\hline No. of cases & 4 & 44 \\
\hline Marker & RASSF1A & DYS \\
\hline Actual cffDNA values & $60.2 \pm 9.7-$ controls & $51.5(31.1-84.9)-$ controls \\
\hline Estimated DR $(95 \% \mathrm{Cl})$ & $119.6 \pm 52.2-$ cases & $71.2(30.3-107.4)-$ cases \\
\hline Estimated AUC $(95 \% \mathrm{Cl})$ & $75 \%(30.0-95.4)$ & $20.5 \%(11.1-34.5)$ \\
\hline P-value & $0.750(0.412-1.00)$ & $0.636(0.539-0.773)$ \\
\hline Power at $5 \%$ type l error $[\%]$ & 0.105 & 0.005 \\
\hline
\end{tabular}

cffDNA - cell free fetal DNA; SD - standard deviation; DR — detection rate; $\mathrm{Cl}$ - confidence interval; AUC — area under the curve

Table 3. Comparison of predictive values for PE of single markers and levels and MoM values of DSCR3, RASSF1A, and HYP2 in the first trimester. Data are expressed as median (interquartile range)

\begin{tabular}{|c|c|c|c|c|c|c|c|c|}
\hline & \multirow{2}{*}{ AUC $(95 \% \mathrm{Cl})$} & \multirow{2}{*}{ PVALUE } & \multicolumn{2}{|c|}{ DETECTION RATE } & \multicolumn{2}{|l|}{ CONTROLS $n=53$} & \multicolumn{2}{|l|}{$P E n=14$} \\
\hline & & & $5 \% \mathrm{FPR}$ & $10 \%$ FPR & Copies $/ \mathrm{mL}$ & MoM & Copies/mL & MoM \\
\hline DSCR3 & $0.563(0.435-0.685)$ & 0.4499 & 16.2 & 23.0 & $\begin{array}{l}890.9 \\
(538.9-1,530.0)\end{array}$ & $\begin{array}{l}1.000 \\
(0.605-1.717)\end{array}$ & $\begin{array}{l}939.1 \\
(685.5-1,718.2)\end{array}$ & $\begin{array}{l}1.054 \\
(0.769-1.929)\end{array}$ \\
\hline RASSF1A & $0.617(0.489-0.734)$ & 0.1358 & 15.0 & 30.1 & $\begin{array}{l}445.6 \\
(262.9-936.4)\end{array}$ & $\begin{array}{l}0.912 \\
(0.538-1.916)\end{array}$ & $\begin{array}{l}668.8 \\
(455.5-961.6)\end{array}$ & $\begin{array}{l}1.369 \\
(0.932-1.968)\end{array}$ \\
\hline HYP2 & $0.682(0.557-0.790)$ & 0.0268 & 32.8 & 45.2 & $\begin{array}{l}5,188.1 \\
(2,042.6-7,682.5)\end{array}$ & $\begin{array}{l}0.921 \\
(0.363-1.364)\end{array}$ & $\begin{array}{l}7,169.6 \\
(4,895.2-12,384.1)\end{array}$ & $\begin{array}{l}1.273 \\
(0.869-2.198)\end{array}$ \\
\hline
\end{tabular}

On the other hand, Silver et al., also used RASSF1A selective amplification and found no association between cffDNA in the first trimester and $\mathrm{PE}$, although these authors did not detect cffDNA in the majority of cases and its assessment was not a primary desired outcome of their study, thereby the preparation process was suboptimal [26].

Kim etal. [6], presented an interesting implementation of the RASSF1A gene. Quantification of DSCR3 and RASSF1A as markers of cffDNA and the HYP2 gene as CfDNA was performed and incorporated into a contingent model. CfDNA as a single marker appeared to be more useful than cffDNA quantification in PE prediction (Tab.3), although low DR of all of these single markers implies validity of its application only after adding other factors.

Poon et al. [27], analyzed a cffDNA quantification method implemented in commercially available screening tests, i.e. chromosome-selective sequencing of non-polymorphic and polymorphic loci, where fetal alleles differ from maternal alleles. As a result, assessment of fetal and maternal cfDNA levels was unlikely to predict subsequent PE (12.9 and 13.3 for $P E$ and normal pregnancies, respectively) and maternal plasma levels of cffDNA at 11-13 weeks of gestation were increased, alongside markers of placental development, and decreased by maternal weight.
In order to adjust values to maternal characteristics and gestational age, a different mode of quantification was presented by Rolnik et al. [28], where total cell-free and fetal fractions were converted to multiples of median (MoM). At 11-13 weeks of gestation, there was a significant increase in total cfDNA $(2.104 \mathrm{GE} / \mathrm{mL}$ vs. $1.590 \mathrm{GE} / \mathrm{mL}$ ) and decrease in median fetal fraction measured by SeqFF (6.8\% vs. $8.7 \%)$, although the differences faded after MoM calculations (controls - 0.963 and 1.037; early PE - 1.199 and 0.993; late PE 1.138 and 0.969, total and fetal fraction, respectively).

Thurik at el. [29], also converted first-trimester cffDNA values to MoM, failing to present predictive values for PE. These authors sought to determine the association between the adjusted cffDNA levels with the so-called 'great obstetrical syndromes' - preeclampsia, pregnancy-induced hypertension, gestational diabetes mellitus and spontaneous preterm birth, with quantification only in the male fetuses as a main disadvantage of the study. The MoM values were lower in women who later developed PIH (pregnancy induced hypertension), but not PE. Alongside many other studies, also in their research cffDNA levels were negatively associated with BMI and smoking, yet positively with PAPP-A. 


\section{SUMMARY}

Over the last decades, second-trimester aneuploidy screening has been displaced by the first-trimester complex approach. It is highly probable that non-invasive prenatal testing (NIPT) with the use of cffDNA will be incorporated into the routine 11-13-week scan. The emerging need for prevention rather than treatment of perinatal complications implies broadening of first-trimester screening. Detailed analysis brought new, mostly incidental applications of NIPT. Circulating tumor DNA (ctDNA) has the ability to distort the reading of the test. Altered genetic profiles were associated with increased incidence of maternal malignancy [30], including ovarian cancer (OC), which could improve the OC screening process [31].

Moreover, Bennett et al., proposed to use NIPT for detecting BRCA1/2 mutations [32], starting a holistic model of insight into the maternal and fetal genome.

In most of the presented studies, no association between first-trimester cffDNA and subsequent development of preeclampsia was confirmed. Heterogeneity of the enrolled groups and multiple types of assessment methods make it difficult to draw clear conclusions. Moreover, most of the studies were based on older quantifying methods, dependent on fetal sex, and large-scale research should be planned, including implementation of massively parallel sequencing and digital analysis of selected regions [16, 33]. It is more likely that those instruments would answer the question whether deviation in first-trimester cffDNA concentration could predict preeclampsia. Also, the majority of the researchers implemented markers not used in commercially available tests, which implies additional assays and does not fulfill the principles of screening per se.

The presented data could lead to the hypothesis that first-trimester cffDNA levels are strongly dependent on the extent of placentation and maternal characteristics (such as volume of distribution related to BMI). Conversely, in late pregnancy, elevated cffDNA levels might be the result of impaired cell turnover and progressive placental insufficiency, leading to hypoxia and subsequent augmented apoptosis.

A contingent model integrating maternal characteristics and cffDNA values is yet another approach, which may be used for PE screening, as low fetal fraction is related to many PE risk factors, such as high median maternal BMI [28]. The abovementioned conclusions indicate a great need of large-scale surveys, which will take into consideration distribution, methods of analysis, maternal characteristics, sample consistency, and subsequent perinatal results. This approach could identify the'low fetal fraction' group and its impact on pregnancy management. Hopefully, the future will bring the answer to the question whether low or very high fetal fraction values, or even failed results, are indications for intensified supervision, such as aspirin implementation or active chromosomal abnormality search.

\section{REFERENCES:}

1. Lo YM, Corbetta N, Chamberlain PF, et al. Presence of fetal DNA in maternal plasma and serum. Lancet. 1997; 350(9076): 485-487, doi: 10.1016/S0140-6736(97)02174-0, indexed in Pubmed: 9274585.

2. Lo YM, Lau TK, Zhang J, et al. Increased fetal DNA concentrations in the plasma of pregnant women carrying fetuses with trisomy 21. Clin Chem. 1999; 45(10): 1747-1751, indexed in Pubmed: 10508120.

3. Gil MM, Quezada MS, Revello R, et al. Analysis of cell-free DNA in maternal blood in screening for fetal aneuploidies: updated meta-analysis. Ultrasound Obstet Gynecol. 2015; 45(3): 249-266, doi: 10.1002/uog.14791, indexed in Pubmed: 25639627.

4. Kagan KO, Sroka F, Sonek J, et al. First-trimester risk assessment based on ultrasound and cell-free DNA vs combined screening: a randomized controlled trial. Ultrasound Obstet Gynecol. 2018; 51(4): 437-444, doi: 10.1002/uog.18905, indexed in Pubmed: 28925570.

5. Wataganara T, Metzenbauer M, Peter I, et al. Placental volume, as measured by 3-dimensional sonography and levels of maternal plasma cell-free fetal DNA. Am J Obstet Gynecol. 2005; 193(2): 496-500, doi: 10.1016/j.ajog.2004.12.015, indexed in Pubmed: 16098876.

6. O'Gorman N, Wright D, Rolnik DL, et al. Study protocol for the randomised controlled trial: combined multimarker screening and randomised patient treatment with ASpirin for evidence-based PREeclampsia prevention (ASPRE). BMJ Open. 2016; 6(6): e011801, doi: 10.1136/bmjopen-2016-011801, indexed in Pubmed: 27354081.

7. Roberge S, Nicolaides K, Demers S, et al. The role of aspirin dose on the prevention of preeclampsia and fetal growth restriction: systematic review and meta-analysis. Am J Obstet Gynecol. 2017; 216(2): 110-120. e6, doi: 10.1016/j.ajog.2016.09.076, indexed in Pubmed: 27640943.

8. Rolnik DL, Wright D, Poon LCY, et al. ASPRE trial: performance of screening for preterm pre-eclampsia. Ultrasound Obstet Gynecol. 2017; 50(4): 492-495, doi: 10.1002/uog.18816, indexed in Pubmed: 28741785.

9. Kosinski P, Sarzynska-Nowacka U, Fiolna M, et al. The practical use of acetylsalicylic acid in the era of the ASPRE trial. Update and literature review. Ginekol Pol. 2018; 89(2): 107-111, doi: 10.5603/GP.a2018.0018, indexed in Pubmed: 29512816.

10. Alberry MS, Maddocks DG, Hadi MA, et al. Quantification of cell free fetal DNA in maternal plasma in normal pregnancies and in pregnancies with placental dysfunction. Am J Obstet Gynecol. 2009; 200(1): 98.e1-98.e6, doi: 10.1016/j.ajog.2008.07.063, indexed in Pubmed: 19121662.

11. Contro E, Bernabini D, Farina A. Cell-Free Fetal DNA for the Prediction of Pre-Eclampsia at the First and Second Trimesters: A Systematic Review and Meta-Analysis. Mol Diagn Ther. 2017; 21(2): 125-135, doi: 10.1007/s40291-016-0245-9, indexed in Pubmed: 27838884.

12. Lau TW, Leung TN, Chan LYS, et al. Fetal DNA clearance from maternal plasma is impaired in preeclampsia. Clin Chem. 2002; 48(12): 2141-2146, indexed in Pubmed: 12446469.

13. Chan KC, Ding C, Gerovassili A, et al. Hypermethylated RASSF1A in maternal plasma: A universal fetal DNA marker that improves the reliability of noninvasive prenatal diagnosis. Clin Chem. 2006; 52(12): 2211-2218, doi: 10.1373/clinchem.2006.074997, indexed in Pubmed: 17068167.

14. Salvianti F, Inversetti $A$, Smid M, et al. Prospective evaluation of RASSF1A cell-free DNA as a biomarker of pre-eclampsia. Placenta. 2015; 36(9): 9961001, doi: 10.1016/j.placenta.2015.07.003, indexed in Pubmed: 26183647.

15. Jani J, Rego de Sousa MJ, Benachi A. Cell-free DNA testing: how to choose which laboratory to use? Ultrasound Obstet Gynecol. 2015; 46(5): 515-517, doi: 10.1002/uog.15733, indexed in Pubmed: 26300279.

16. Chiu RWK, Lo YM. Noninvasive prenatal diagnosis empowered by high-throughput sequencing. Prenat Diagn. 2012; 32(4): 401-406, doi: 10.1002/pd.3822, indexed in Pubmed: 22467171.

17. Ashoor G, Syngelaki A, Poon LCY, et al. Fetal fraction in maternal plasma cell-free DNA at 11-13 weeks' gestation: relation to maternal and fetal characteristics. Ultrasound Obstet Gynecol. 2013; 41(1): 26-32, doi: 10.1002/uog.12331, indexed in Pubmed: 23108725.

18. Revello R, Sarno L, Ispas A, et al. Screening for trisomies by cell-free DNA testing of maternal blood: consequences of a failed result. Ultrasound Obstet Gynecol. 2016; 47(6): 698-704, doi: 10.1002/uog.15851, indexed in Pubmed: 26743020.

19. Rolnik DL, da Silva Costa F, Lee TJ, et al. Association between fetal fraction on cell-free DNA testing and first-trimester markers for pre-eclampsia. UItrasound Obstet Gynecol. 2018; 52(6): 722-727, doi: 10.1002/uog.18993, indexed in Pubmed: 29318732.

20. Wang E, Batey A, Struble C, et al. Gestational age and maternal weight effects on fetal cell-free DNA in maternal plasma. Prenat Diagn. 2013; 33(7): 662-666, doi: 10.1002/pd.4119, indexed in Pubmed: 23553731. 
21. Wright $D$, Wright $A$, Nicolaides $K H$. A unified approach to risk assessment for fetal aneuploidies. Ultrasound Obstet Gynecol. 2015; 45(1): 48-54, doi: 10.1002/uog.14694, indexed in Pubmed: 25315809.

22. Gil MM, Accurti V, Santacruz B, et al. Analysis of cell-free DNA in maternal blood in screening for aneuploidies: updated meta-analysis. Ultrasound Obstet Gynecol. 2017; 50(3): 302-314, doi: 10.1002/uog.17484, indexed in Pubmed: 28397325.

23. Norton M, Musci T, Wapner R. 358: Relationship between 1st trimester fetal fraction of cell-free DNA from maternal plasma and preeclampsia in a large general pregnancy population. American Journal of Obstetrics and Gynecology. 2015; 212(1): S188, doi: 10.1016/j.ajog.2014.10.404.

24. Sifakis $S$, Zaravinos A, Maiz N, et al. First-trimester maternal plasma cell-free fetal DNA and preeclampsia. Am J Obstet Gynecol. 2009; 201(5): 472.e1-472.e7, doi: 10.1016/j.ajog.2009.05.025, indexed in Pubmed: 19631923.

25. Papantoniou N, Bagiokos V, Agiannitopoulos K, et al. RASSF1 A in maternal plasma as a molecular marker of preeclampsia. Prenat Diagn. 2013; 33(7): 682-687, doi: 10.1002/pd.4093, indexed in Pubmed: 23526657.

26. Room TB, Lauderdale F, Goldberg IJ. HHS Public Access. 2015; 12(2): $130-40$.

27. Poon LCY, Musci T, Song $\mathrm{K}$, et al. Maternal plasma cell-free fetal and maternal DNA at 11-13 weeks' gestation: relation to fetal and maternal characteristics and pregnancy outcomes. Fetal Diagn Ther. 2013; 33(4): 215-223, doi: 10.1159/000346806, indexed in Pubmed: 23466432.

28. Rolnik DL, O'Gorman N, Fiolna M, et al. Maternal plasma cell-free DNA in the prediction of pre-eclampsia. Ultrasound Obstet Gynecol. 2015; 45(1): 106-111, doi: 10.1002/uog.14671, indexed in Pubmed: 25252010.
29. Thurik FF, Lamain-de Ruiter M, Javadi A, et al. Absolute first trimester cell-free DNA levels and their associations with adverse pregnancy outcomes. Prenat Diagn. 2016; 36(12): 1104-1111, doi: 10.1002/pd.4940, indexed in Pubmed: 27734510.

30. Bianchi DW, Chudova D, Sehnert AJ, et al. Noninvasive Prenatal Testing and Incidental Detection of Occult Maternal Malignancies. JAMA. 2015; 314(2): 162-169, doi: 10.1001/jama.2015.7120, indexed in Pubmed: 26168314.

31. Kulasingam V, Diamandis EP, Cohen PA, et al. Abnormal plasma DNA profiles in early ovarian cancer using a non-invasive prenatal testing platform: implications for cancer screening. BMC Med. 2016; 14(1): 126, doi: 10.1186/s12916-016-0667-6, indexed in Pubmed: 27558279.

32. Bennett J, Chitty L, Lewis C. Non-invasive Prenatal Diagnosis for BRCA Mutations - a Qualitative Pilot Study of Health Professionals' Views. J Genet Couns. 2016; 25(1): 198-207, doi: 10.1007/s10897-0159858-0, indexed in Pubmed: 26174937.

33. Palomaki GE, Kloza EM, Lambert-Messerlian GM, et al. DNA sequencing of maternal plasma to detect Down syndrome: an international clinical validation study. Genet Med. 2011; 13(11): 913-920, doi: 10.1097/GIM.0b013e3182368a0e, indexed in Pubmed: 22005709.

34. Gratacós E, Nicolaides K. Clinical perspective of cell-free DNA testing for fetal aneuploidies. Fetal Diagn Ther. 2014; 35(3): 151-155, doi: 10.1159/000362940, indexed in Pubmed: 24931002.

35. Kim MJ, Kim SY, Park SY, et al. Association of fetal-derived hypermethylated RASSF1 A concentration in placenta-mediated pregnancy complications. Placenta. 2013;34(1):57-61, doi: 10.1016/j.placenta.2012.11.001, indexed in Pubmed: 23187089. 